\title{
Temporal Memory of Interfood Interval Distributions with the Same Mean and Variance
}

\author{
Russell M. Church and Donna M. Lacourse
}

\begin{abstract}
Brown University
The time of occurrence of reinforced lever responses of rats depends on the characteristics of the distribution of times from food to the next available food. Two groups of 10 rats were trained, in counterbalanced order, on two variable-interval schedules of reinforcement that were equated for the mean, standard deviation, and range of the intervals from food to the next available food, but which differed in shape. The differences in the shape of the interfood interval distributions resulted in differences in the distribution of interfood intervals, of postreinforcement pauses, the function relating response rate to time since food, and the power spectra of times of response. Quantitative timing models, such as scalar timing theory and a multipleoscillator model, differ in their assumptions about the nature of the internal clock and the representation of time in memory. The multiple-oscillator model and scalar timing theory accounted for different features of the data. (c) 2001 Academic Press
\end{abstract}

Key Words: random interval; time discrimination; temporal memory.

An animal searching for food must decide where to go and when to go there. This involves movement in both space and time. A temporal search task is one in which the spatial aspects of the task are held constant but the temporal aspects are varied. For example, food may always be found in a single location but at various times. The problem for the animal is when to search for food in that location.

Any time-based schedule of reinforcement involving a single operant response may be defined as a temporal search task. Such a food schedule may be characterized as a distribution of intervals between the time that food occurs and the time that the next food is available. The first response after food is made available results in food delivery.

This research was supported by the National Institute of Mental Health Grant MH44234 to Brown University. Hilary A. Broadbent contributed to the development of some of the methods of data analysis used in this article; Kimberly Kirkpatrick provided useful comments on the manuscript. The raw data (time of occurrence of each stimulus, response, and reinforcement) are available at http://www.Brown.edu/Research/Timelab.

Address correspondence and reprint requests to Russell M. Church, Department of Psychology, Box 1853, Brown University, Providence RI 02912. Fax: (401) 863-1300. E-mail: Russell_Church@Brown.edu. 
At one extreme of predictability, each food may be made available at a fixed interval after the previous food delivery, so that an animal with the ability to time perfectly would be able to predict exactly the time of availability of the next food. Behavior under conditions of fixed-interval responding has been studied extensively, and timing theories are able to account for many of the characteristics of performance (Church \& Broadbent, 1990; Gibbon \& Church, 1984; Killeen \& Fetterman, 1988).

At the other extreme of predictability, each food can be made available at a random interval after the previous food delivery. In a random-interval schedule of reinforcement, food is made available after a random exponential waiting time with some mean. In this schedule, the conditional probability of food becoming available is independent of the time since the last food delivery, so that an animal with the ability to time perfectly would have no ability to predict the time of occurrence of the next food availability. Of course, a time sense would still be useful for making estimates of the mean time from food until the next food is made available, which could be reflected in the mean response rate. The mean response rate has been found to be well described as a decreasing hyperbolic function of the mean interval between the delivery of food and the next available food (Herrnstein, 1970). In the remainder of this article, "food" refers to the time of food delivery and "prime" refers to the time of food availability. These are the scheduled and actual times of reinforcement, respectively. The sequence of food-prime intervals is the scheduled reinforcement series, and the sequence of foodfood intervals is the actual reinforcement series.

Performance is markedly affected by characteristics of the distribution of food to prime intervals, such as the mean and variance. The mean time of starting to respond on a fixed-interval schedule of reinforcement is proportional to the interval from food to prime; the standard deviation of the start times is also proportional to this interval; thus, the coefficient of variation (the standard deviation divided by the mean) is relatively constant (Church, Meck, \& Gibbon, 1994; Gibbon, 1977). The mean time of starting to respond also depends on the variability of the food-prime interval (Brunner, Fairhurst, Stolovitzky, \& Gibbon, 1997; Church, Lacourse, \& Crystal, 1998). The observation that animals respond sooner after reinforcement if the time to the next reinforcement is more variable may be related to the preference for variable over fixed times of reinforcement (for example, Davison, 1969; Gibbon, Church, Fairhurst, \& Kacelnik, 1988).

There is also some evidence that performance may be affected by the shape of the distribution, independently of its mean and variance. Although there are many differences in the performance of rats on fixed versus variable schedules of reinforcement, the effects of different distributions of the variable interval are less certain. Comparisons have been made between uniform and exponential distributions of intervals with the same mean (Catania \& Reynolds, 1968). Other comparisons have been made between forward and 
backward Exponential distributions (Brunner, Fairhurst, Stolovitszky, \& Gibbon, 1997; Gibbon, Church, Fairhurst, \& Kacelnik, 1988). Conclusions from these data are qualified by the small effects often observed and by the difficulty of isolating, with these particular pairs of distributions, the shape of the distribution from other factors such as the range of possible times.

The present experiment is concerned with the determinants of responding under conditions in which the scheduled distributions of food-prime intervals have the same mean, variance, and range, but different distribution forms. Thus, if the behavior of the animal were determined by the mean interval, the global predictability of the interval, or by the extreme values, the behavior under the two schedules of reinforcement would be the same. But if the behavior of the rats is affected by the form of the distribution, then the behavior may not be determined by such simple summary measures as a measure of central tendency, a measure of variability, or a percentile. An effect of the difference in distribution forms alone would suggest that the behavior was determined by the local predictability of the occurrence of food. The empirical purpose of the present experiments was to determine how temporal search performance differs in an environment in which food occurs over the same range of possible times with a fixed mean time $(60 \mathrm{~s})$ and a fixed standard deviation (60 s), but with differing distribution forms.

In one condition, food was made available at time determined by an Exponential distribution (a random waiting-time distribution) with a mean and standard deviation of $1 \mathrm{~min}$. In the other condition, food was made available at times determined by a Wald (inverse Gaussian) distribution with a mean and standard deviation of $1 \mathrm{~min}$. The forms of these distributions are shown in Fig. 1 (see Evans, Hastings, \& Peacock, 1993). The top panel shows the probability density functions (with areas of 1.0); the middle panel shows the distribution functions, the probability of food being available by any given time after the previous food; and the bottom panel shows the hazard functions, the conditional probability of food being made available at any particular time following the delivery of food. Although the two distributions were equated for overall mean and standard deviation, they differed with respect to local predictability of the time of occurrence of food availability.

The theoretical purpose of the experiment was to determine the extent to which timing theories can account for any observed differences in the response patterns. Quantitative timing models, such as scalar timing theory (Gibbon, Church, \& Meck, 1984) and a multiple-oscillator, connectionist model (Church \& Broadbent, 1990), differ in their assumptions about the perceptual representation of time, temporal memory, and the basis for decisions about time. The perceptual representation of time in scalar timing theory is a single pacemaker that sends pulses to an accumulator; in the multiple-oscillator model, a set of oscillators' half-phases serve as the perceptual representation of time. The memory representation used in scalar timing theory is a distribution of transformed accumulator values (an exem- 

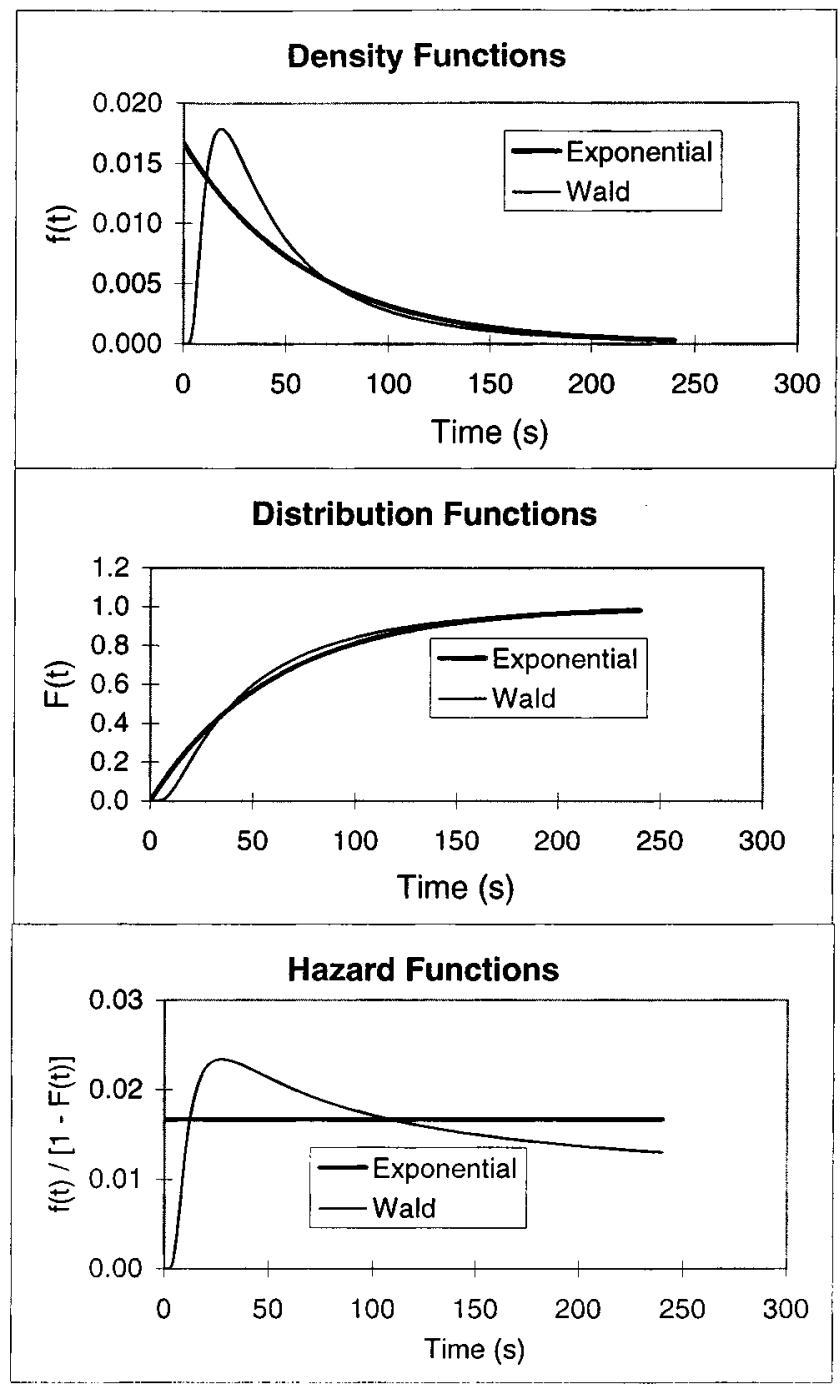

FIG. 1 Exponential and Wald distributions, each with a mean and standard deviation of $60 \mathrm{~s}$. The top panel shows the density functions, the middle panel shows distribution functions, and the bottom panels shows the hazard functions.

plar memory); the memory representation in the multiple-oscillator model is a linear combination of autoassociation matrices of perceived times (a prototype memory). The decision process in scalar timing theory consists of a comparison of a random sample of a single value from a temporal memory distribution to the current value in the accumulator; the decision process in 
the multiple-oscillator model consists of a comparison of the current perceptual representation with the entire temporal memory matrix. On the basis of data currently available, it is not possible to determine which of these fundamentally different sets of assumptions about temporal perception, memory, and decision is more likely to provide the basis for the development of a quantitative theory of timing that will fit many different descriptive measures of behavior obtained in many different procedures (Church, 1997a, 1997b).

\section{METHOD}

Animals

Twenty experimentally naive male albino Sprague-Dawley viral antibody-free rats (Charles River Breeding Laboratory, Kingston, NY) were used. They were 49 days old on arrival and housed in individual cages. After 1 week of $a d$ lib food and water, they were fed $15 \mathrm{~g} /$ day. Water was continuously available both in the home cages and in the operant boxes in the testing room. The colony room was on a 12:12 light:dark cycle. During the dark phase (from 8:45 AM until 8:45 PM), dim red lights illuminated the colony room, and dim red lights were present at all times in the testing room. Sessions began about 9 AM and 11:15 AM each day.

\section{Apparatus}

Ten identical operant boxes $(23 \times 30 \times 30 \mathrm{~cm})$ were equipped with a lever (Med Associates ENV-112BX, $4.8 \times 1.9 \mathrm{~cm}$ wide and $6 \mathrm{~cm}$ above the floor) $3.5 \mathrm{~cm}$ to the left of a food reservoir $(5 \times 5 \times 2 \mathrm{~cm})$. A pellet dispenser (Med Associates ENV-203) delivered 45-mg Noyes (Improved Formula A) pellets into the food cup. A panel opposite the lever and food cup had a water bottle mounted outside the box. Each box was in a ventilated, sound-attenuating chamber $(74 \times 38 \times 60 \mathrm{~cm}$ high $)$. Two Gateway 486 DX2/66 computers running Med-PC Medstate Notation Version 2.0 controlled experimental events and recorded the time at which each event and lever press occurred with 10 -ms resolution.

\section{Procedure}

Pretraining began when the rats were 109 days old. It consisted of three sessions in which each lever press was followed by a food pellet. A session ended when the rat made 60 responses or after $60 \mathrm{~min}$, whichever came first. The number of rats that made 60 responses in $60 \mathrm{~min}$ on Sessions 1, 2, and 3 was 8,13 , and 19 , respectively. (The remaining rat was given a fourth session of training and made 60 responses on that session.)

The rats were randomly divided into two groups of 10 rats each. In Phase I (Sessions 1-30) the Exponential distribution was used for the determination of times of food availability for one group and the Wald distribution was 
used for the other group. In Phase 2 (Sessions 31-60) these conditions were reversed. The daily sessions were two hours in length and began at 9:30 AM and 11:45 AM. The session began with the onset of the houselight and the insertion of the lever into the box.

The Exponential density function is $f(t)=(1 / b) e^{-t / b}$, where $t$ is the time from 0 to infinity and $b$ is the mean (top panel of Fig. 1). The Exponential distribution function is $F(t)=1-e^{(-t / b)}$ (middle panel of Fig. 1). The equation that was used for selecting random values from this distribution was $t$ $=-b \ln (r)$, where $r$ is a uniform random number between 0 and 1. A distinctive feature of the Exponential distribution is its constant hazard function at the level of $1 / b$ (bottom panel of Fig. 1). The mean parameter, $b$, was set to $60 \mathrm{~s}$. The Exponential distribution may arise from a process in which the probability of an event is a constant (Luce, 1986).

The Wald density function is also defined from $t=0$ to infinity; it has two parameters representing central tendency and variability; the mean and standard deviation were set to $60 \mathrm{~s}$. A table of 1000 times (to the nearest $0.1 \mathrm{~s})$ was prepared, corresponding to probabilities of $0.001,0.002, \ldots$, 0.999 of the Wald distribution function. These times varied from 4.8 to 501.3 $\mathrm{s}$. Random values from the Wald distribution functions were obtained by using a uniform random number between 0.001 and 0.999 (multiplied by 1000) as an index to this table of times that was sampled with replacement. The Wald distribution may arise from a random walk with one absorbing barrier (Luce, 1986). Unlike the Exponential distribution, the hazard function for the Wald distribution is not constant. The hazard function rises quickly to around $30 \mathrm{~s}$ and then falls gradually, so that there is local predictability.

At the beginning of the session, and following each delivery of food, a randomly selected time from the appropriate distribution was determined as the time for the next food to be primed. This food was delivered immediately following the first lever press after the prime. Lever presses before the prime were recorded, but had no effects.

\section{Data Analysis}

A record was made of the times of each experimenter-controlled event (such as priming food, delivering food, and turning on a light) and each ratcontrolled event (such as pressing or releasing the lever and licking the water bottle). The time-event codes that were analyzed in this report are the times of each lever response, priming of food, and delivery of food. The four major dependent variables were (a) the distribution of food-food intervals, (b) the distribution of intervals from food to next response (the postreinforcement pauses), (c) the response rate as a function of time since food, and (d) the power spectrum of response times based on 10-ms intervals for each 2-h session. If a response occurred in a 0.01-s interval, a " 1 ', was recorded; otherwise a " 0 " was recorded. Power spectra were calculated with this sequence. Power spectra were also calculated on a transformation of this se- 
quence that was previously used by Broadbent (1994). A half-fill transform was used to fill the time between successive responses: The first half of the interval between successive $1 \mathrm{~s}$ was replaced by $+1 \mathrm{~s}$ in each 0.01 -s unit of time and the second half was replaced by $-1 \mathrm{~s}$ in each 0.01 -s unit of time. The power spectra were calculated using the Matlab power spectral density (psd) function, with a length of 16,384 intervals $\left(2^{14}\right)$ and linear detrending, but no filtering and no overlap. Maxima and minima of these functions were identified as the maxima and minima of a 16th-order polynomial, which provided a smooth approximation of the spectrum.

Results for the food-food and food-prime distributions are based on all 30 sessions under each condition; results for the postreinforcement pause, the response rate, and the spectral analysis are based on the last 10 sessions under each condition. Medians were used to combine measures across sessions of a given rat; means were used to combine measures across rats.

\section{RESULTS}

The distribution of food-prime (scheduled) intervals in the Exponential and Wald conditions are shown in Fig. 2; they approximate the form of the theoretical density functions shown in the top panel of Fig. 1. The distributions of the food-food intervals received by the rats in the Exponential and Wald conditions are shown by the data points in Fig. 2. In both the Exponential and Wald conditions there was a rightward shift in the food-food times compared to the food-prime- this is wasted time. The shift was fairly uniform in the Wald, but there was some evident bias in the Exponential condition. There was an overabundance of food deliveries at about 5 and $26 \mathrm{~s}$.

Figure 3 shows the mean probability of the occurrence of the first response after food as a function of time since food. The mean time of the first response after food occurred earlier in the Exponential than in the Wald condition during Sessions $21-30$ (5.44 and $11.67 \mathrm{~s}), t(18)=7.9, P<.001$. This difference was also observed after the conditions were reversed during Sessions 51-60, (5.67 and $10.98 \mathrm{~s}), t(18)=4.9, P<.001$. The standard deviation of the first response after food was lower in the Exponential than in the Wald condition during Sessions 21-30 (4.44 and 10.74 s), $t(18)=8.6, P$ $<.001$. This difference was also observed after the conditions were reversed during Sessions 51-60 (4.71 and 9.70 s), $t(18)=4.8, P<.001$.

The mean probability of a response as a function of time since food is shown in Fig. 4 for rats in the Exponential and Wald conditions. In the Exponential condition, there was a rapid rise followed by a decrease to a fairly steady rate; in the Wald condition, there was a more gradual rise and then a decrease to a relatively steady rate that was at a level similar to that of the Exponential. The time of the maximum response rate occurred earlier in the Exponential than in the Wald condition, both during Sessions 21-30, $t(18)$ $=3.6, P<.001$, and after the conditions were reversed during Sessions $51-$ $60, t(18)=3.8, P<.001$. 
Exponential
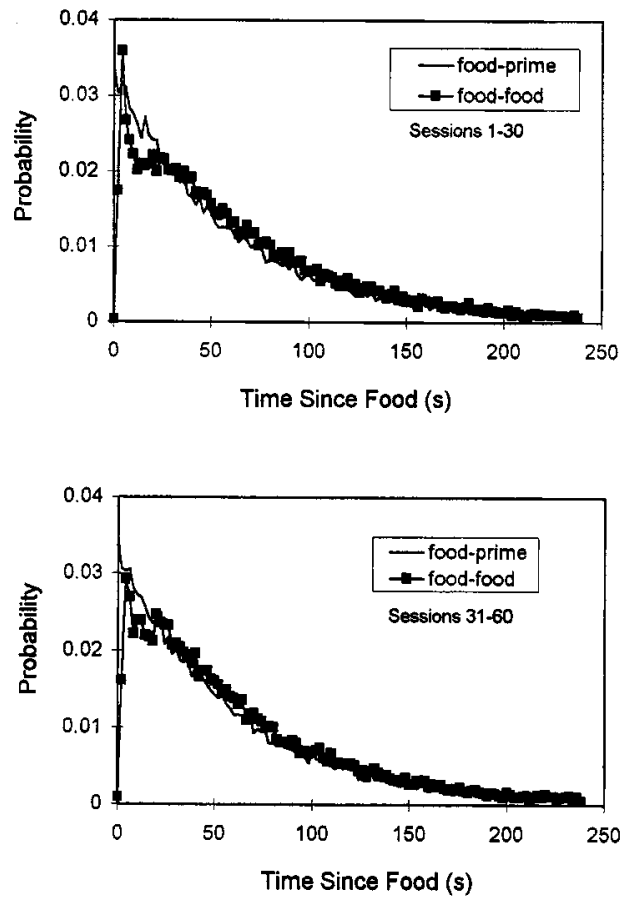

Wald
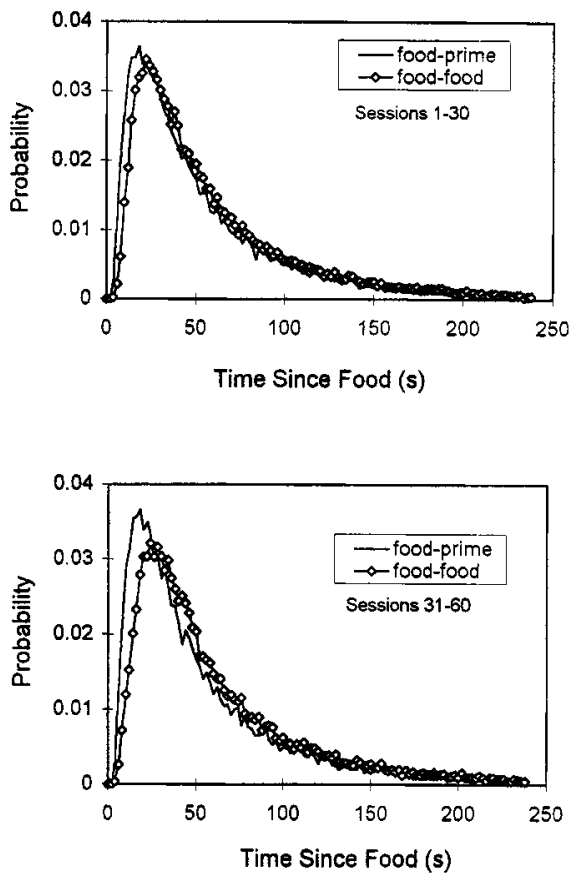

FIG. 2 The mean probability of food becoming available (the food-prime interval) and of food being delivered (the food-food interval) as a function of time since food delivery. The left panels shows the distributions for the Exponential condition, and the right panels show the distribution for the Wald condition.

The power spectrum for one rat in the Exponential Condition on Sessions 51-60 is shown in Fig. 5. A standard power spectrum analysis is reported for the data in the top left panel of Fig. 5. The power simply decreases as a function of frequency without any indication of maximum power at some intermediate frequency. This is contrasted with the power of an exponential waiting time distribution with the same mean interresponse interval as the observed data (labeled "random" in the top left panel of Fig. 5).

The top right panel of Fig. 5 shows a power spectrum analysis of the same data with the half-filled transformation (see "Data Analysis"). With this transformation the power does increase to a maximum at some intermediate frequency. This occurs both with the data and with an exponential waiting time distribution with the same mean interresponse interval as the observed data, although the locations of the maxima are different.

The bottom panels replot this information as a difference between the data and the simulation (labeled "Data-Random"). This difference between the 

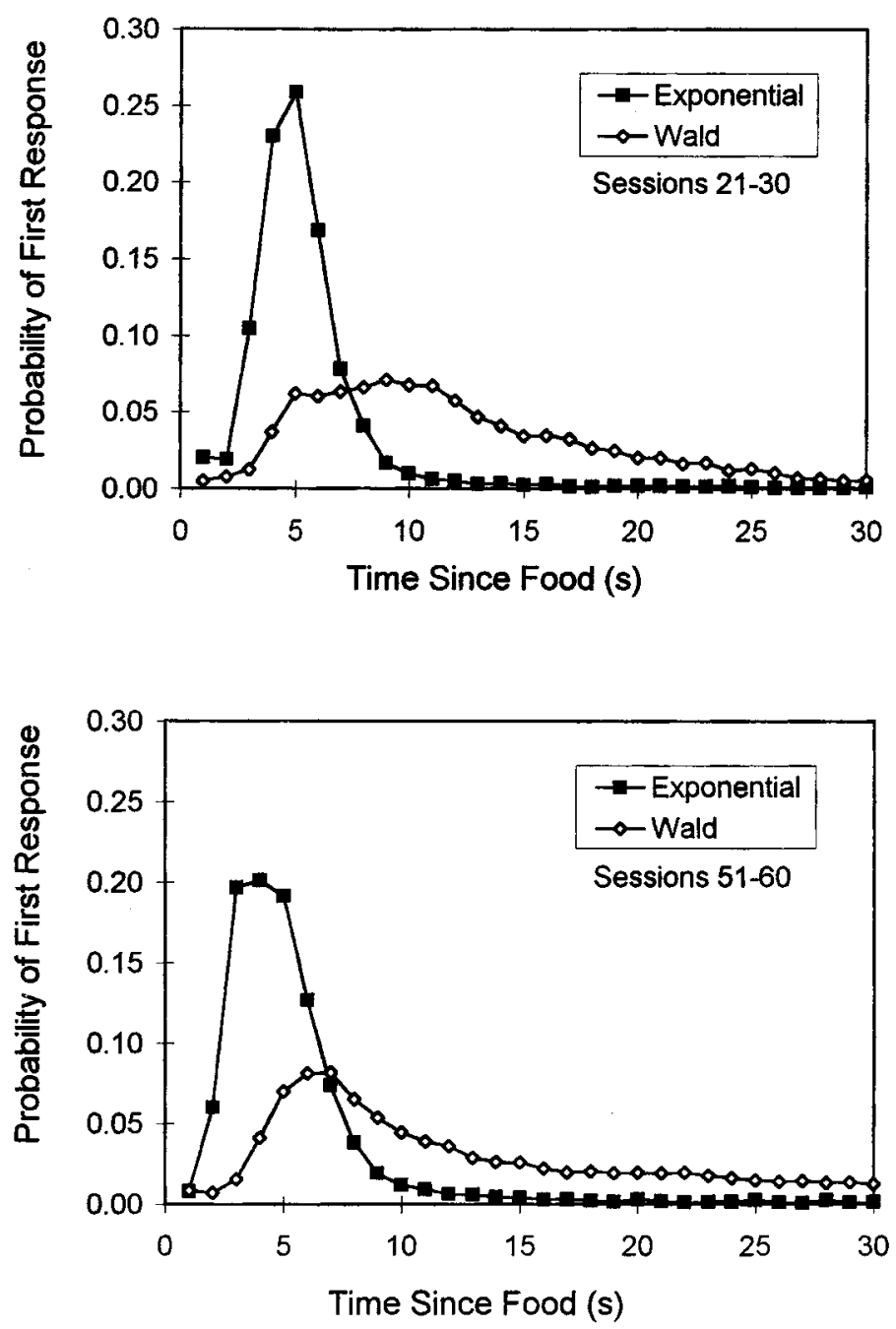

FIG. 3 The mean probability that the first response after food occurs in each 1-s interval since food. The last 10 sessions of the first and second phases are shown for the Exponential and Wald distributions.

power spectrum of the data and of the exponential waiting time with the same mean interresponse interval as the observed data is referred to as "Relative Power." Without a transformation, the relative power was high at low frequencies and decreased at higher frequencies. With the half-filled transformation, the shape of the relative power was quite different. There was a maximum near a frequency of $0.014 \mathrm{~Hz}$ and a minimum near a frequency of $0.048 \mathrm{~Hz}$. 

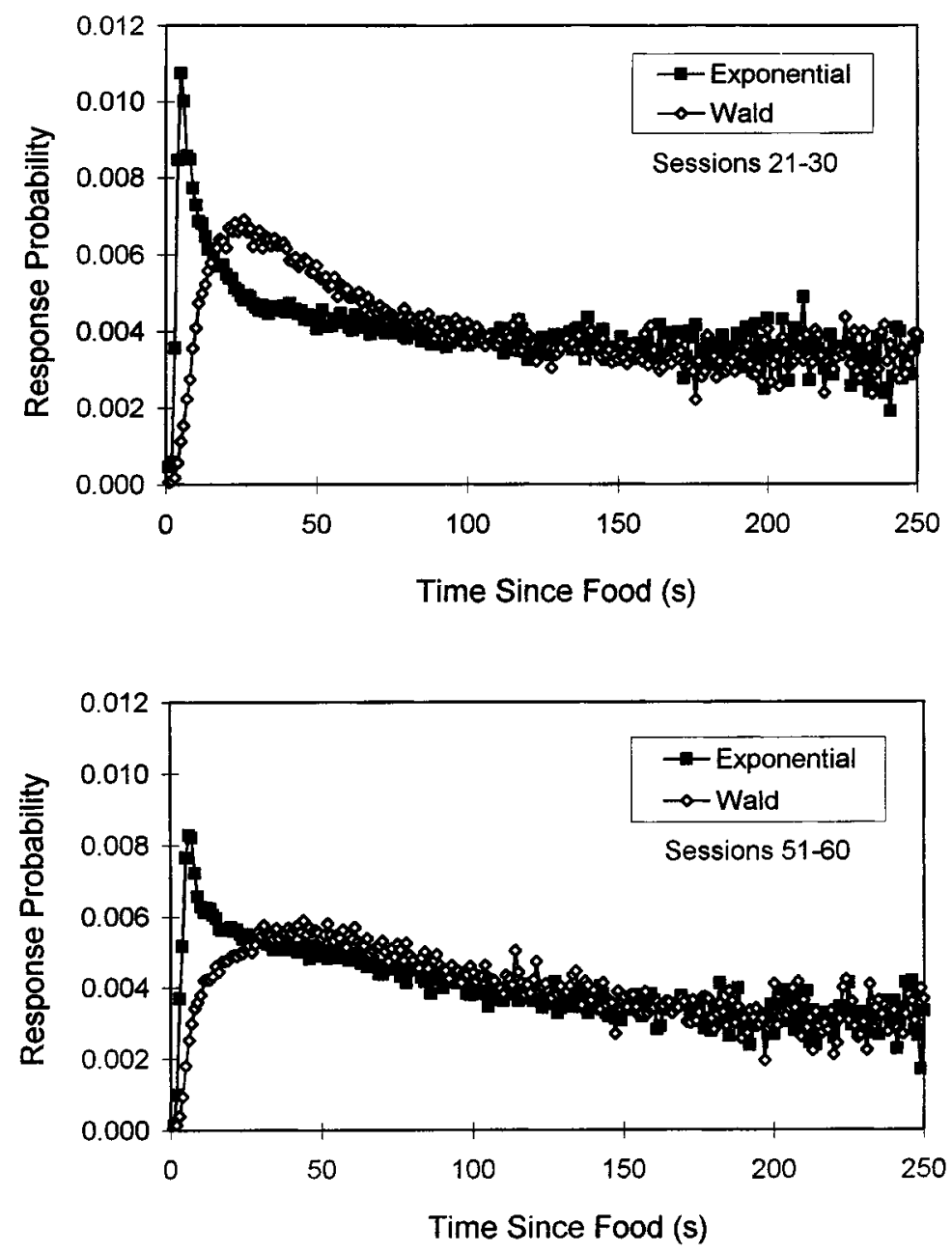

FIG. 4 The mean probability of a response in each 1-s interval since food. The last 10 sessions of the first and second phases are shown for Exponential and Wald distributions.

The general response pattern of the rat in Fig. 5 was characteristic of the mean relative power spectra of rats with the Exponential and Wald distribution during Sessions 51-60, as shown in Fig. 6. Without a transformation, the relative power decreased with frequency; with the half-fill transformation, the relative power had a maximum and, perhaps, also a minimum. There was a maximum near a frequency of $0.015 \mathrm{~Hz}$ and a minimum near a frequency of $0.121 \mathrm{~Hz}$ in the Exponential condition and a minimum near a frequency of 0.027 and $0.078 \mathrm{~Hz}$ in the Wald condition. 

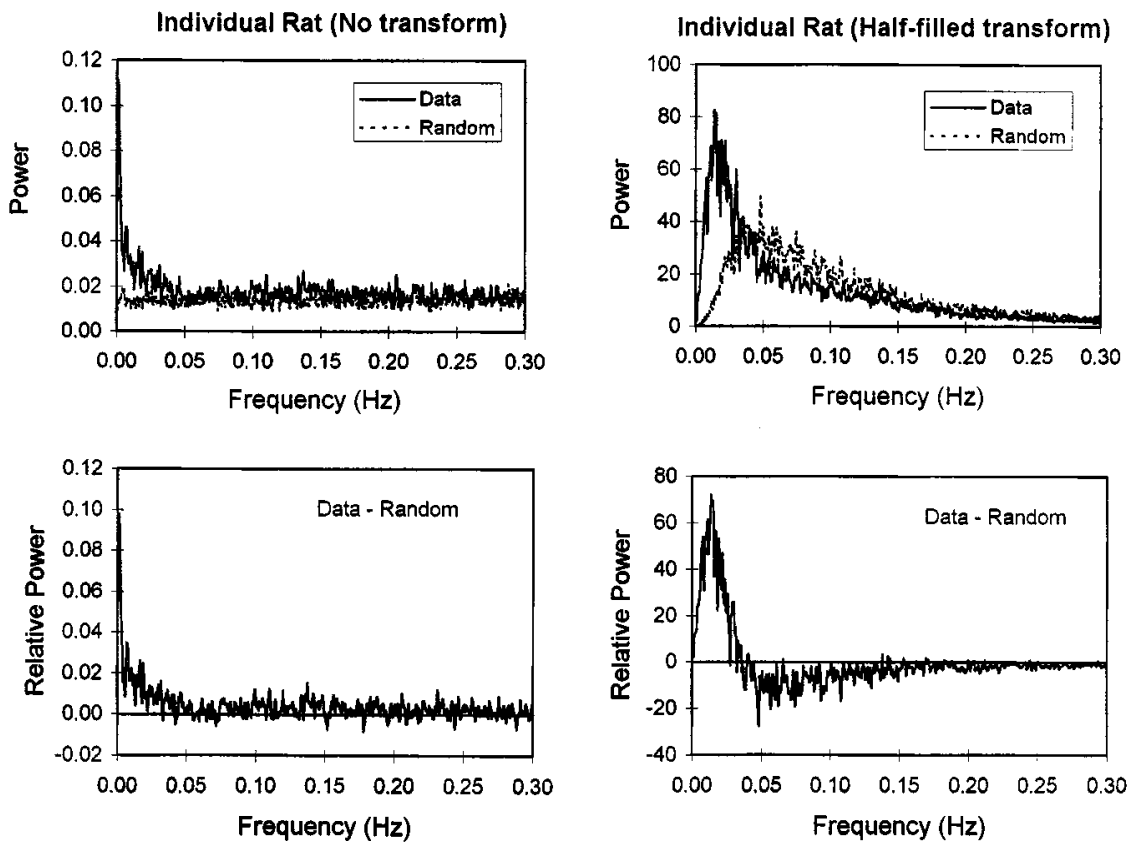

FIG. 5 Power spectra for the interresponse intervals for one rat in the Exponential condition during the last 10 sessions of the second phase. The top left panel shows the power spectrum of the data for a sample with an exponential waiting time distribution with the same mean. Bottom left panels show the relative power (the difference between the two functions in the top panel). The panels on the right are the same analyses based on the half-filled transform.

\section{DISCUSSION}

Previous research described in the introduction has shown that temporal search depends on the mean, standard deviation, and range of the distribution of times between an event and the availability of the next food. The present experiment provides further evidence that the behavior of rats is also influenced by the distribution form, even when mean and standard deviation are held constant. The two distribution functions used in this experiment (center panel of Fig. 1) appear to be quite similar, but differences between them are emphasized by the density (top panel) and hazard functions (bottom panel). For example, the Exponential density function is higher than the Wald density function in the first $10 \mathrm{~s}$ and lower from 11 to $55 \mathrm{~s}$ (top panel of Fig. 1). These differences were sufficient to produce substantial differences in the behavior of the rats as shown by the distribution of food-food intervals (Fig. 2), distribution of times of postreinforcement pauses (Fig. 3), and the response rate as a function time since food (Fig. 4).

The distribution of food-food intervals in the Wald condition appeared to be displaced by a few seconds to the right of the distribution of food-prime 
Exponential (No transform)

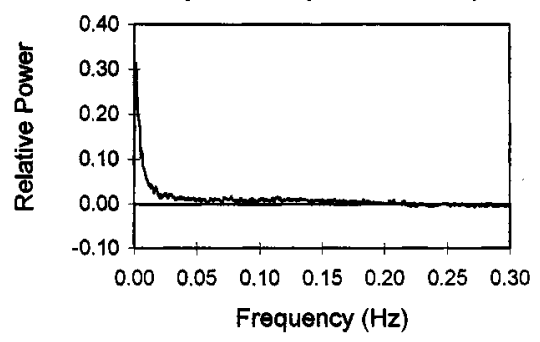

Wald (No transform)

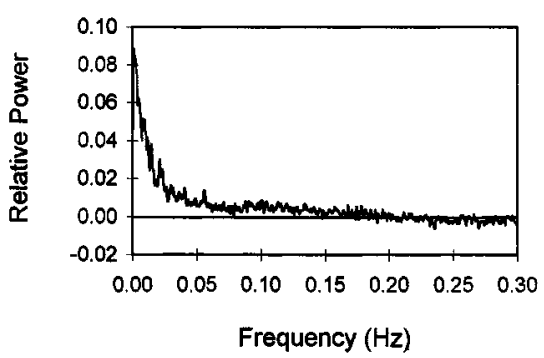

Exponential (Half-filled transform)

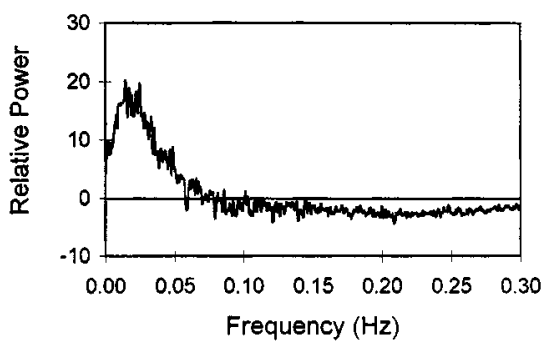

Wald (Half-filled transform)

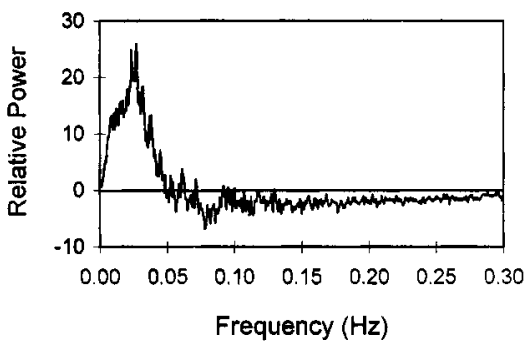

FIG. 6 The mean relative power as a function of response frequency (in hertz). The left panels are without a transformation, and the right panels are with the half-filled transform; the top panels are for the Exponential condition and the bottom panels are for the Wald condition. Data from Sessions 51-60.

intervals. This is consistent with a short interval between the availability of food and the response required to deliver the food. In contrast, the Exponential condition was not simply a rightward displacement of the distribution of times from food to prime (Fig. 2). Compared to the scheduled food-prime interval, the actual food-food interval was initially low, with two distinct maxima (at about 5 and $26 \mathrm{~s}$ ). This result, previously reported for the Exponential distribution (Broadbent, 1994) and for the Uniform distribution of intervals with a minimum of $0 \mathrm{~s}$ but not of $30 \mathrm{~s}$ (Church, Lacourse, \& Crystal, 1998), provides evidence for a nonmonotonic process initiated by food. The initial low probability of a lever response after food presumably reflects competition with the consummatory act of eating the food; the first maximum may represent the increased responding due to the increasing probability of a reinforcement following a period without responding. Such a process could lead to a damped oscillation in which two local maxima are clearly evident. This process would occur whenever the reinforcement density is high immediately after food (as in the Exponential distribution and the Uniform distribution with a minimum of zero) and not under conditions in which the reinforcement density is low immediately after food (as in the Wald distribution and the Uniform distribution with a minimum of $30 \mathrm{~s}$ ). 
The postreinforcement pause may represent, in part, a reaction to the food presentation: Some fixed time might be required for consumption of the food followed by other activities, such as grooming or drinking. But this does not account for the large differences in the postreinforcement pause of rats in the Exponential and Wald conditions (Fig. 3). The pause was shorter in the Exponential than in the Wald condition presumably because the reinforcement density was initially higher shortly after reinforcement.

The response rate as a function of time since food provided a rough correspondence to the density, distribution, and hazard functions of the two conditions. The response rate was initially higher in the Exponential condition, then higher in the Wald condition, and finally about equal in the two conditions (Fig. 4).

The spectral density functions, however, did not discriminate between the two conditions. Without the transform, the relative power decreased as a function of frequency. With a half-fill transform the spectral density functions had at least one clear maximum, as reported by Broadbent (1994). The location of the maximum of these spectral functions does not serve as a measure of a dominant period because they are affected by the mean response rate and a maximum also occurs with a random input.

Theories of timing that have been developed to account for performance on fixed intervals may be directly extended to apply to variable intervals. Each of the theories may be characterized by assumptions about the perception of a time interval, the memory about a time of reinforcement, and a decision about whether to respond (Church \& Kirkpatrick, 2000).

According to scalar timing theory (Gibbon \& Church, 1984; Gibbon, Church, \& Meck, 1984), a single pacemaker sends pulses to an accumulator which is the perceived time since reinforcement. When reinforcement occurs, the perceived time, perhaps transformed, is stored as an example in reference memory; the accumulator is reset; and a random example from reference memory is retrieved. At each small unit of time, the perceived time is compared to the retrieved memory according to a ratio comparison. If that ratio is smaller than a threshold that is sampled from a normal distribution of possible threshold values, a response will be made.

According to a multiple-oscillator model of timing (Church \& Broadbent, 1990), the perceived time since reinforcement is the vector of half-phases of 11 oscillators with $d$ periods of $1,2,4,8, \ldots, 1024 \mathrm{~s}$. When reinforcement occurs, the perceived time is sent to an autoassociation matrix (working memory) that is combined with the remembered time in the reference memory matrix according to a linear combination rule and the oscillators are reset. At each small unit of time, the perceived time is compared to the product of the perceived time and reference memory, and, if the cosine is small relative to a threshold, a response will be made.

Simulation of the multiple-oscillator model was based on the version described by Church and Broadbent (1990), with a coefficient of variation of 

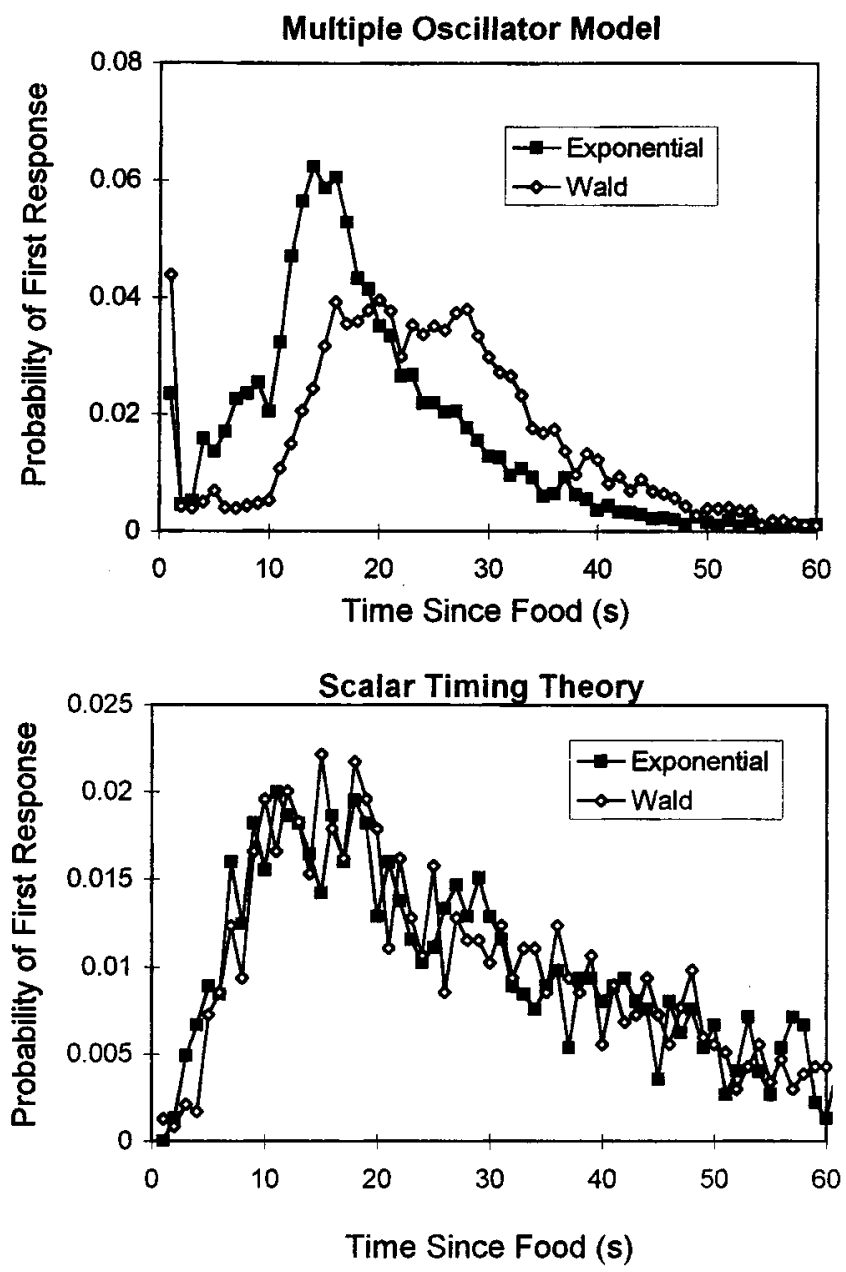

FIG. 8 Simulation of the mean probability of the first response after food in each 1-s interval since food delivery for the Exponential and Wald conditions. The top panel is from the multiple-oscillator model; the bottom panel is from the scalar timing model. (Compare to results shown in Fig. 3.)

the asymptotic performance of the multiple-oscillator model leads to local irregularities (Church, Lacourse, \& Crystal, 1998; Crystal, Church, \& Broadbent, 1997). The overall form of the food-food interval distribution produced by the scalar timing model approximated the data less closely.

With the multiple-oscillator model, the distributions of the postreinforcement pauses in the Exponential and Wald conditions were different: The postreinforcement pauses of the rats in the Exponential condition were earlier (Fig. 8), as was observed in the data (Fig. 3). The simulated mean postrein- 
forcement pause was greater than the observed pause, so the correspondence was qualitative rather than quantitative. With the scalar timing model the distributions of the postreinforcement pauses in the Exponential and Wald conditions were similar, although they would presumably not be identical because more short interfood intervals would be in memory in the Exponential condition than in the Wald condition.

With the multiple-oscillator model, the mean response rate increased more rapidly in the Exponential condition than the Wald condition and then decreased to an asymptote that was similar to the rat data (Fig. 9). These fea-
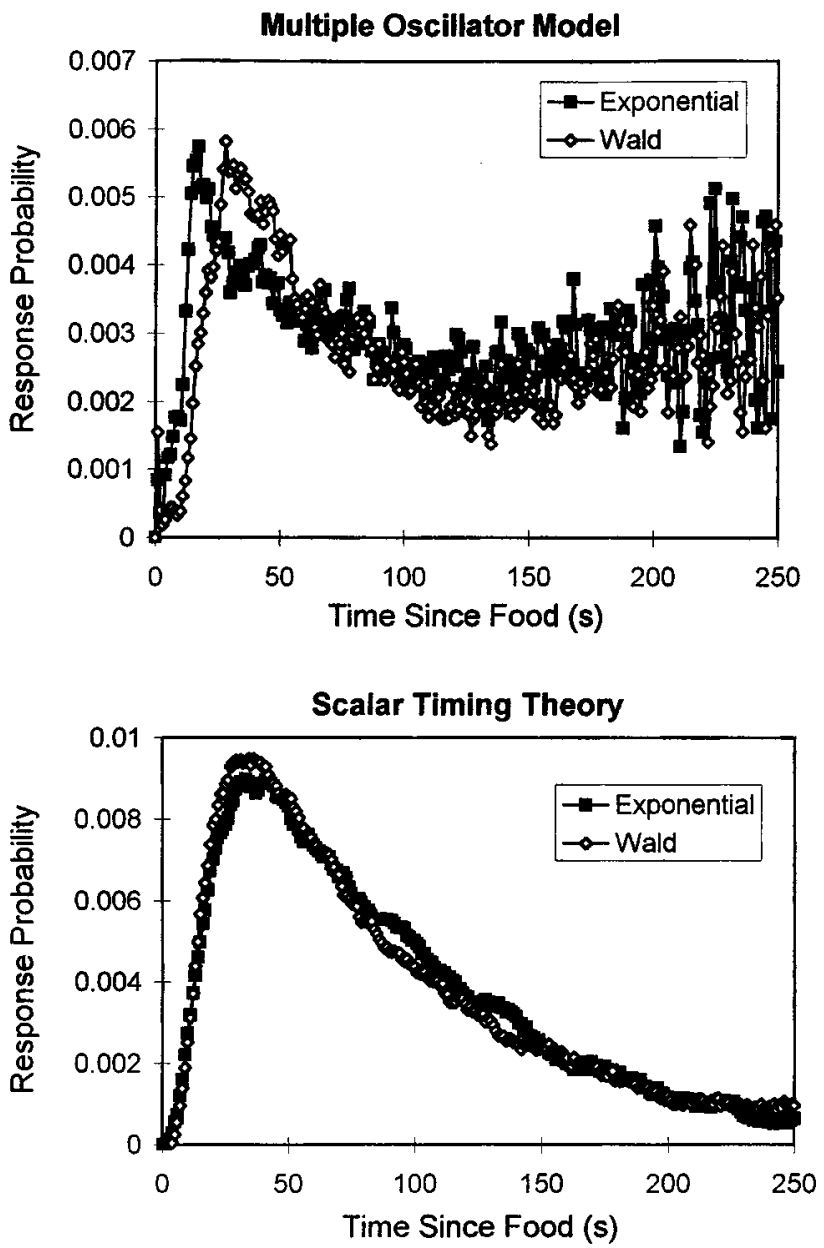

FIG. 9 Simulation of the mean probability of a response in each 1-s interval since food delivery for the Exponential and Wald conditions. The top panel is from the multiple-oscillator model; the bottom panel is from scalar timing theory. (Compare to results shown in Fig. 4.) 
Multiple Oscillator Model
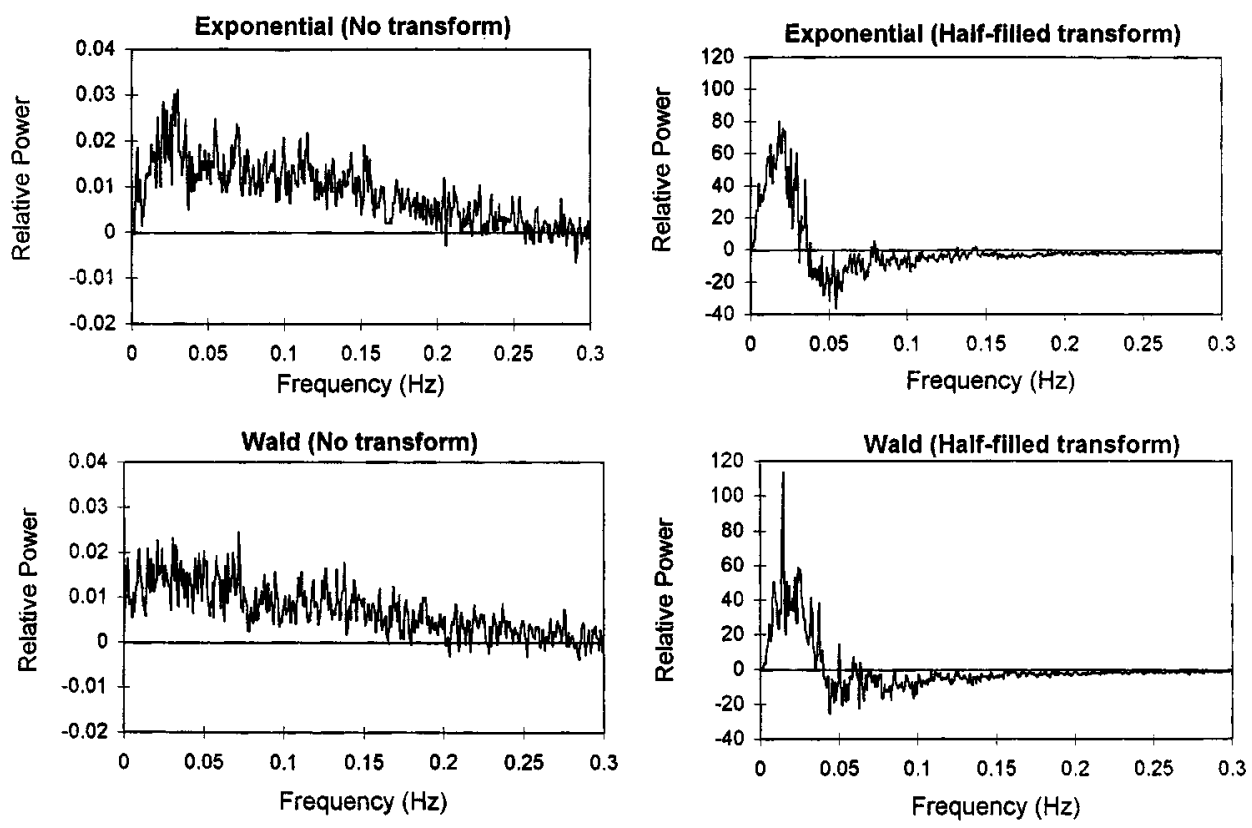

FIG. 10 Simulation with multiple-oscillator model of the relative power as a function of response rate for the Exponential (top panels) and Wald conditions (bottom panels) with the untransformed data (left panels) and the half-filled transformed data (right panels). (Compare to results shown in Fig. 6.)

tures were observed in the data (Fig. 4). With the scalar timing model, the response rate functions in the two conditions were similar and the shape was different from that observed in the data.

The spectral power produced by the multiple-oscillator model is shown in Fig. 10. These results may be compared to those shown in Fig. 6. In contrast to the data, the simulated relative power of the untransformed data in both the Exponential and the Wald conditions increased to a maximum and then decreased to approximately zero (Fig. 10, left panels). The relative power of the transformed data in both the Exponential and Wald conditions increased to a maximum, decreased below zero, and then returned to approximately zero (Fig. 10, right panels). Thus, the multiple-oscillator model produced a qualitative fit to the power spectra of the transformed data, but it failed to produce a qualitative fit to the power spectra of the untransformed data.

The spectral power produced by the scalar timing model is shown in Fig. 11. These results may be compared to those shown in Fig. 6. The relative power of the untransformed data in both the Exponential and the Wald condi- 
Scalar Timing Theory
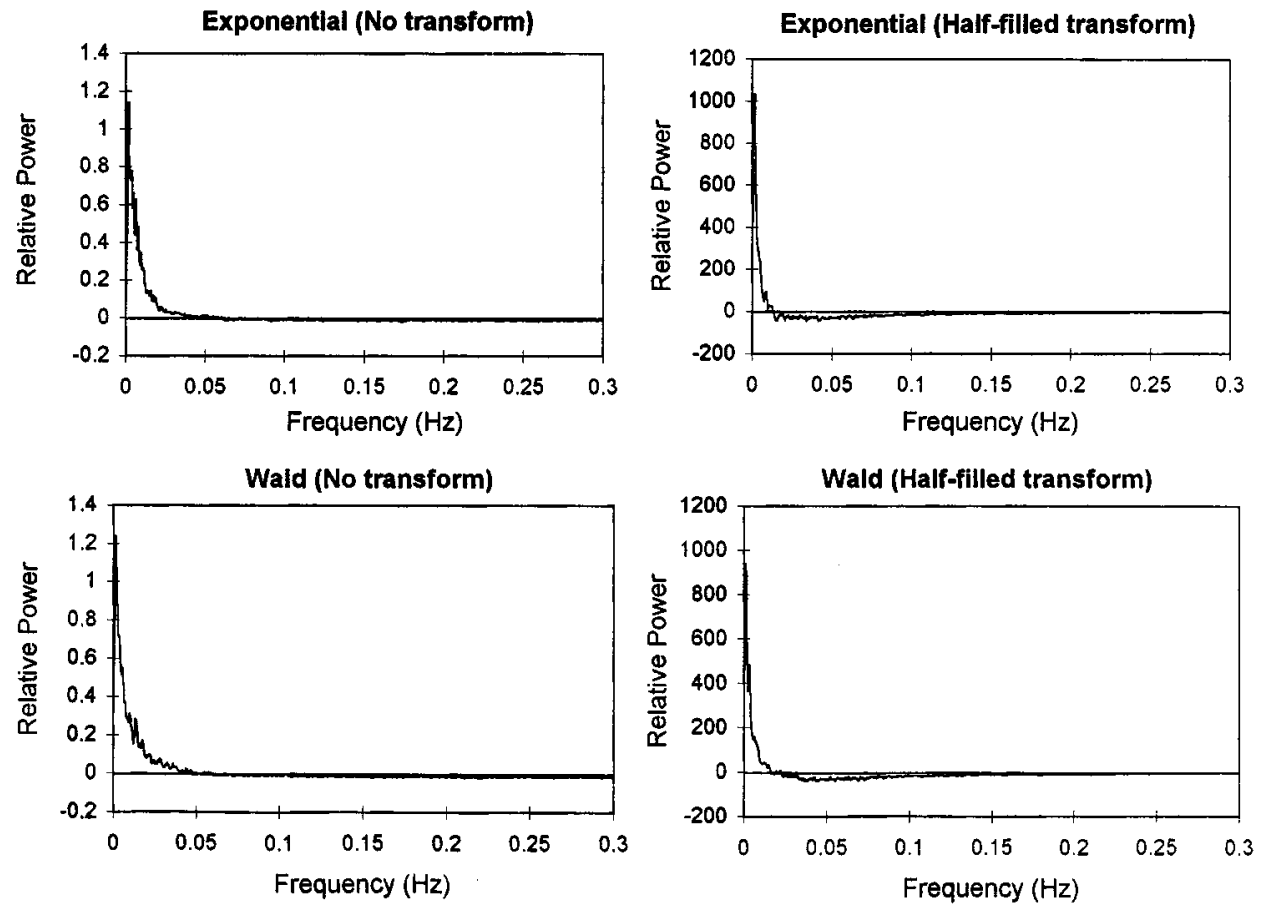

FIG. 11 Simulation with scalar timing model of the relative power as a function of response rate for the Exponential (top panels) and Wald conditions (bottom panels) with the untransformed data (left panels) and the half-filled transformed data (right panels). (Compare to results shown in Fig. 6.)

tion was high at low frequencies and gradually decreased to approximately zero (Fig. 11, left panels). The relative power of the transformed data in both the Exponential and Wald conditions was high at low frequencies, gradually decreased below zero, and then returned to approximately zero (Fig. 10, right panels). Thus, the scalar timing theory produced a qualitative fit to the power spectra of the untransformed data, but it failed to provide a qualitative fit to the power spectra of the transformed data.

Although theories of timing have been relatively successful in dealing with procedures involving fixed intervals, they have had more difficulty in accounting for behavior in procedures involving variable intervals. The multiple-oscillator model has been used to provide qualitative fits to the behavior of rats in random interval schedules of reinforcement (Broadbent, 1994) and to uniform schedules of reinforcement (Church, Lacourse, \& Crystal, 1998). In the present experiment it also provided qualitative fits to many, although 
not all, of the response measures examined. Probably the most essential feature of this model is its assumption that animals retrieve a representation of the entire distribution of experienced times of reinforcement from memory. In contrast, the usual assumption in scalar timing theory is that animals retrieve a single example of a previously experienced time of reinforcement from memory.

Scalar timing theory did not provide qualitative fits to most of the response measures examined. It is possible that the standard version of the theory that applies to fixed intervals does not apply to variable intervals (Brunner, Fairhurst, Stolovitzky, \& Gibbon, 1997). The proposal is that, in a fixed interval schedule, the animal takes a single random sample from memory of a reinforced time, but in a variable interval schedule, the animal takes two samples. One of these is taken from an early percentile and is used for starting a high response rate; the other is taken from a later percentile and is used for ending that high response rate. This modification increases the effect of different distribution shapes. Of course, it is necessary to specify the conditions under which the animal uses the one-sample and two-sample versions of the theory.

Ideally, the same theory of timing would apply to different procedures and to different response measures. If different models are required for different procedures, then the model must include an additional process that permits the animal to classify procedures. If different models are required for different ways to describe a time series of responses, then it is necessary to recognize that they are models of specific dependent variables rather than models of the generating function.

At present, no theory of timing provides a quantitative fit of multiple response measures from different variable interval schedules. Further modifications of timing theories will be necessary to account for the various descriptions of behavior of rats in an environment in which food is available at variable intervals. The fact that the form of the distribution of interfood intervals affects performance means that a timing theory must be based on more than a few summary measures of a distribution of intervals.

\section{REFERENCES}

Broadbent, H. A. (1994). Periodic behavior in a random environment. Journal of Experimental Psychology: Animal Behavior Processes, 20, 156-175.

Brunner, D., Fairhurst, S., Stolovitszky, G., \& Gibbon, J. (1997). Mnemonics for variability: Remembering food delay. Journal of Experimental Psychology: Animal Behavior Processes, 22, 68-82.

Catania, A. C., \& Reynolds, G. S. (1968). A quantitative analysis of the responding maintained by interval schedules of reinforcement. Journal of the Experimental Analysis of Behavior, 11, 327-383.

Church, R. M. (1997a). Quantitative models of animal learning and cognition. Journal of Experimental Psychology: Animal Behavior Processes, 23, 379-389.

Church, R. M. (1997b). Timing and temporal search. In C. M. Bradshaw \& E. Szabadi (Eds). 
Time and behaviour: Psychological and neurobehavioural analyses (pp. 41-78). Amsterdam: Elsevier.

Church, R. M., \& Broadbent, H. A. (1990). Alternative representations of time, number, and rate. Cognition, 37, 55-81.

Church, R. M., \& Kirkpatrick, K. (2000). Theories of conditioning and timing. In R. R. Mowrer \& S. B. Klein (Eds.), Handbook of contemporary learning theories (pp. 211253). Hillsdale, NJ: Erlbaum.

Church, R. M., Lacourse, D. M., \& Crystal, J. D. (1998). Temporal search as a function of the variability of interfood intervals. Journal of Experimental Psychology: Animal Behavior Processes, 24, 291-315.

Church, R. M., Meck, W. H., \& Gibbon, J. (1994). Application of scalar timing theory to individual trials. Journal of Experimental Psychology: Animal Behavior Processes, 20, $135-155$.

Crystal, J. D., Church, R. M., \& Broadbent, H. A. (1997). Systematic nonlinearities in the memory representation of time. Journal of the Experimental Analysis of Behavior: Animal Behavior Processes, 23, 267-282.

Davison, M. C. (1969). Preference for mixed-interval versus fixed-interval schedules. Journal of the Experimental Analysis of Behavior, 12, 247-252.

Evans, M., Hastings, N., \& Peacock, B. (1993). Statistical distributions (2nd ed.). New York: Wiley.

Gibbon, J. (1977). Scalar expectancy theory and Weber's law in animal timing. Psychological Review, 84, 279-325.

Gibbon, J., \& Church, R. M. (1984). Sources of variance in an information processing theory of timing. In H. L. Roitblat, T. G. Bever, \& H. S. Terrace (Eds.) Animal cognition. Hillsdale, NJ: Erlbaum.

Gibbon, J., Church, R. M., Fairhurst, S., \& Kacelnik, A. (1988). Scalar expectancy theory and choice between delayed rewards. Psychological Review, 95, 102-114.

Gibbon, J., Church, R. M., \& Meck, W. H. (1984). Scalar timing in memory. In J. Gibbon \& L. Allan (Eds.), Annals of the New York Academy of Sciences: Volume 423. Timing and time perception (pp. 52-77). New York: The New York Academy of Sciences.

Herrnstein, R. J. (1970). On the law of effect. Journal of the Experimental Analysis of Behavior, 13, 243-266.

Killeen, P. R., \& Fetterman, J. G. (1988). A behavioral theory of timing. Psychological Review, 95, 274-295.

Luce, R. D. (1986). Response times: Their role in inferring elementary mental organization. New York: Oxford Univ. Press.

Received August 2, 2000

Revised November 1, 2000 